\title{
PLAN DE MARKETING PARA LA EXPORTACIÓN DE PANETÓN A ESTADOS UNIDOS PERIODO 2012 - 2013
}

\author{
MARKETINGPLANFOR EXPORTING PANETTONE TOTHE UNITED STATESFROM THE \\ YEAR 2012 to 2013
}

Ernesto Altamirano Flores ${ }^{1}$ y Luis Espinoza Villanueva ${ }^{2}$

\begin{abstract}
Resumen
En el Perú las empresas panetoneras de Lima metropolitana, no sólo producen panetón en su mayoría, también producen toda las variedades de panes y pasteles, se enfrentan a un mercado nacional altamente competitivo con las empresas trasnacionales e informales. Por tanto, el objetivo principal de esta investigación es, elaborar un plan de marketing para la exportación de panetón producido en Lima, con la finalidad de incrementar las ventas, posicionar el producto en el mercado internacional y ser rentable en el desarrollo de la actividad, donde se tiene como mercado objetivo, al consumidor de Los Ángeles - California en Estados Unidos. La metodología utilizada fue Descriptiva - Exploratoria y se realizó bajo un diseño No Experimental. Antes de establecer el Plan de Marketing se han desarrollado cuatro fases de análisis de resultados, que buscan obtener respuesta a las variables: ventas, posicionamiento y rentabilidad. Los resultados obtenidos en las cuatro fases de estudio permitieron tener las herramientas necesarias para la generación de la propuesta de un plan de marketing para ingresar al mercado de Estados Unidos, donde la principal estrategia de marketing para el ingreso al mercado externo, es la genérica de Liderazgo en costos.
\end{abstract}

Palabras clave: Plan de Marketing, Panetón, Estados Unidos, Exportación

\begin{abstract}
In Peru, the panettone or panetón making companies of the metropolitan area of Lima do not only produce panetón as a main product, but they also produce all varieties of bread sand cakes. They face highly competitive market with transnational corporations and informal markets. The main goal of this research is to develop a marketing plan for the export of Panettone produced in Lima. This will result in increased sales, product positioning in the international market and be profitable in the development of the activity. The target market in the US is the consumers in Los Angeles, California. The methodology used was descriptive exploratory and was conducted under a non-experimental design

Prior to establishing the marketing plan, they developed four stages of results, seeking answers to the following variables, sales, positioning, and profitability. The results obtained in the four phases of the study allowed us to have the necessary tools for generating a proposal of a marketing plan to enter the US market. The main marketing strategy for entering the foreign market is to initiate the leadership in the cost.
\end{abstract}

Key words: Marketing Plan, Panettone, United States, Export

\section{Introducción}

El panetón o panettone, es el más célebre postre navideño que no tiene una historia clara ni única de cómo se dio a conocer alrededor del mundo. Algunas de ellas, están relacionadas con el amor, son de Milán, la ciudad en cuyos hornos nació esta dulce preparación, tiene la grata fama de ser uno de los más románticos del mundo, también hay otras historias que lo relaciona con la religión.

Las empresas productoras de panetones en la ciudad de Lima, tienen como meta contribuir en el crecimiento y búsqueda de oportunidades del mercado externo, este trabajo será beneficioso para las empresas dedicadas al rubro del panetón, este aporte, está basado en la elaboración de plan de marketing, que ayude en la exportación del producto para mejorar las condiciones de vida de los empresarios peruanos.

\section{Objetivo general}

Diseñar un plan de marketing para la exportación y comercialización de panetón en la ciudad de Lima metropolitana.

\section{Objetivos específicos}

Determinar la oferta comercial exportable del panetón de la ciudad de Lima metropolitana.

Evaluar las ventajas competitivas, el concepto de los productos y marcas del panetón.

Analizar la actividad industrial y comercial desarrollada para el panetón, permitiendo mejorar la rentabilidad y generar un plan de marketing. 


\section{A. Marco teórico}

a. Origen de panetón. (AMPEX, 2010).El panetón o panettone, es el más célebre postre navideño que no tiene una historia clara ni única de cómo se dio a conocer alrededor del mundo.

b. Nombres vulgares en otros idiomas. (AMPEX, 2010). Menciona los nombres del panetón en diferentes idiomas de acuerdo al país donde se elabora, como se observa en la Tabla 1.

Tabla 1. Nombres Vulgares En Otros Idiomas

\begin{tabular}{cccc}
\hline País & Nombre común & País & Nombre común \\
\hline EEUU & Sweetbaed & España & $\begin{array}{c}\text { Pan dulce, panetone } \\
\text { o panettone } \\
\text { Panetone, Pan de } \\
\text { Pascua }\end{array}$ \\
Alemania & $\begin{array}{c}\text { Ptollen o Pan de } \\
\text { Cristo }\end{array}$ & Brasil & $\begin{array}{c}\text { Panettone o Pan de } \\
\text { pascua italiano }\end{array}$ \\
Argentina $\begin{array}{c}\text { Pan dulce o Pan } \\
\text { de Navidad } \\
\text { Pan de pascua, } \\
\text { Panetón o } \\
\text { pan de pascua } \\
\text { criollo }\end{array}$ & Benezuelivia & Panetón \\
\hline \multicolumn{3}{c}{ Chile } & Panetón \\
\hline
\end{tabular}

Fuente: Elaboración Propia (2011)

c. Propiedades nutritivas del panetón (AMPEX, 2010) el panetón contiene los ingredientes, como se observa en la Tabla 2.

Tabla 2. Valores Nutricionales Del Panetón

\begin{tabular}{cccc}
\hline \multicolumn{4}{c}{ Informacion nutricional } \\
\hline Contiene & $100 \mathrm{~g}$ & $\begin{array}{c}\text { Porción } \\
(100 \mathrm{~g})\end{array}$ & $\% \mathrm{VRN}\left(^{*}\right)$ \\
\hline Energía (Kcal) & 380 & 380 & $19 \%$ \\
Calorías de la grasa & 140 & 140 & - \\
(Kcal) & 7 & 7 & $14 \%$ \\
Proteína (g) & 15 & 15 & $23 \%$ \\
Grasa (g) & 8 & 8 & $40 \%$ \\
Grasa saturada (g) & 90 & 90 & $30 \%$ \\
Colesterol (mg) & 55 & 55 & $18 \%$ \\
Carbohidratos (g) & 21 & 21 & - \\
Azúcares (g) & 2 & 2 & $8 \%$ \\
Fibra (g) & 120 & 120 & $5 \%$ \\
Sodio (mg) & 48 & 48 & $6 \%$ \\
Vitamina A (ug) & 0 & 0 & $0 \%$ \\
Vitamina C (mg) & 40 & 40 & $5 \%$ \\
Calcio (mg) & 1.3 & 1.3 & $9 \%$ \\
Hierro (mg) & & &
\end{tabular}

Fuente: Nestlé Perú.

\section{B. Marketing}

\section{La estructura del plan de marketing}

Análisis de la situación, Investigación de mercados, Formulación estratégica de marketing, Comprende el establecimiento de las estrategias, Evaluación económica - financiera:

\section{Materiales y métodos}

\section{Materiales}

Documentos de la empresa, Cuestionarios de estimación de producción, Reportes de las empresas industrializadoras de panetón.

\section{Métodos}

- Tipo de investigación. La metodología utilizada es DESCRIPTIVA - EXPLORATORIA, donde se tiene como objetivo indagar la incidencia y los valores que se manifiesta una o más variables.

- Diseño de la investigación. La investigación se ejecutó con el diseño NO EXPERIMENTAL - TRANSVERSAL, debido a que no se modifica deliberadamente variables y se realiza dentro de un tiempo determinado. .

\section{Universo, población y muestra}

- Universo. El universo está representado por el número de empresas productoras de panetón en Lima metropolitana, los cuales están identificados como empresas panificadoras y procesadoras, alcanzando un total de 61, para la campaña 2010.

- Población. La población está representada por todas las empresas panificadoras formales, participantes en la última campaña del 2010, llegando a un total de 61.

- Muestra. El tamaño muestral se ha determinado según la fórmula de muestreo cuando la población es finita realizado por Mayorga y Araujo (2010), los cálculos y parámetros considerados se presentan a continuación.

$$
\mathbf{n}=\frac{Z^{2} \cdot \mathbf{p} \cdot \mathbf{q} \cdot \mathbf{N}}{e^{2} \cdot(\mathbf{N}-\mathbf{1})+Z^{2} \cdot \mathbf{p} \cdot \mathbf{q}}
$$

Dónde:

n: Muestra $=i$ ?

$\mathrm{N}$ : Población $=61$

$Z^{2}$ : Nivel de confianza $(95 \%-1,96)$

$\mathrm{e}^{2}$ : Error permitido $(5 \%)$ o $(0,05)$

p: Proporción de que el evento ocurra en un 0,5 (50\%)

p: Proporción de que el evento no ocurra en un 0,5 (50\%).

$\mathrm{n}=\frac{(1.96)^{2} \times 0.5 \times 0.5 \times 61}{(0.05)^{2}(61-1)+(1.96)^{2} \times 0.5 \times 0.5}$

$\mathrm{n}=\mathbf{5 8 . 5 8 4 4} / \mathbf{1 . 1 1 0 4}=\mathbf{5 3}$ 
a. Procedimiento de análisis de datos. Los resultados del procedimiento de análisis de datos se presentan en las siguientes fases.

PRIMERA FASE: Resultados de la Oferta Comercial Exportable del panetón.

SEGUNDA FASE: Resultados de las Ventajas Competitivas, productos y marcas, como herramientas de gestión para posicionar el producto en el mercado internacional.

TERCERA FASE: Resultados del Análisis de las Actividades Productivas.

CUARTA FASE: Resultados de la Evaluación del Análisis de las encuestas y la interrelación de las variables propuestas.

\section{QUINTA FASE: PLAN DE MARKETING}

\section{Resultados y discusión}

Resultados de la oferta comercial exportable de paneton

\section{A. Análisis de la demanda}

En la tabla 3 Se muestran las exportaciones a los principales mercados a nivel mundial en (dólares EUA Miles), con la partida 1905909000 donde Estados Unidos es el mercado más importante para nuestras exportaciones seguido por Venezuela que en años anteriores ocupaba el primer lugar.

Tabla 3. Exportaciones De Panetón Con La Partida 1905909000

\begin{tabular}{ccccc}
\hline País & 2007 & 2008 & 2009 & 2010 \\
\hline Estados Unidos & 1446 & 1613 & 1376 & 1806 \\
Venezuela & 2296 & 2923 & 1911 & 978 \\
Bolivia & 213 & 490 & 712 & 661 \\
Chile & 187 & 162 & 268 & 332 \\
Otros & 930 & 999 & 788 & 1059 \\
Total & 5072 & 6187 & 5055 & 4836 \\
\hline
\end{tabular}

Fuente: Elaboración Propia (2011). Valores Trade Map.

\section{B. Porcentaje de participacion en el año 2010}

En el Fig. 1: se muestra, que para el año 2010, lidera como destino de las exportaciones del panetón, Estados Unidos superando a Venezuela, que en años anteriores lideraba el destino en las exportaciones de este producto

\section{Análisis de la oferta}

- Producción. La producción nacional de panetón de las marcas registradas en INDECOPI, es 29 millones de unidades para la campaña del año 2010.

- Volumen de las exportaciones peruana. Según datos estadísticos de la SUNAT, el Perú viene exportando panetón con la partida 195909000 desde el año 2007, que son productos producidos en su mayoría en Lima Metropolitana, el volumen de las exportaciones se observan en la Tabla 4.

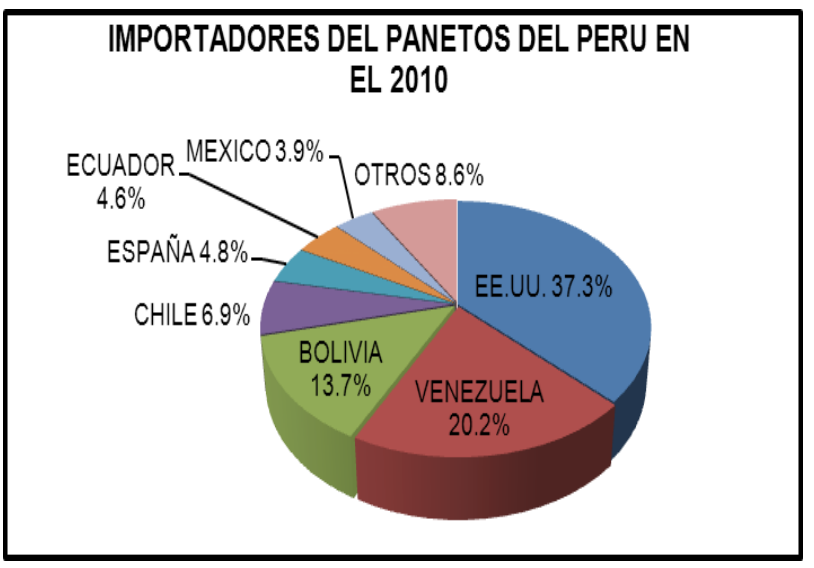

Figura 1. Porcentaje De Participación De Los Países

Fuente: Elaboración: Propia (2010) valores TradeMap

Tabla 4. Exportación De Panetón, Miles Us\$ Fob/Tm

\begin{tabular}{ccccc}
\hline Año & FOB US\$ & $\begin{array}{c}\text { Peso Neto } \\
\text { TM. }\end{array}$ & $\begin{array}{c}\text { Variación } \% \\
\text { FOB }\end{array}$ & $\begin{array}{c}\text { Variación } \\
\text { Volumen \% }\end{array}$ \\
\hline 2007 & 5,072 & $1,573.24$ & & \\
2008 & 6,187 & $1,582.90$ & $22 \%$ & $0.60 \%$ \\
2009 & 5,055 & $1,628.13$ & $-18 \%$ & $2.85 \%$ \\
2010 & 4,836 & $1,518.74$ & $-4 \%$ & $-6.71 \%$ \\
\hline
\end{tabular}

Fuente: SUNAT (2011)

\section{- Mercado nacional de panetón}

Según el análisis del latín panel de Diciembre de 2010 en 14 de las principales ciudades del país, Nestlé ocupa el primer lugar del mercado a través de su marca DONOFRIO, con el 30\%, se muestra en la fig. 2 .

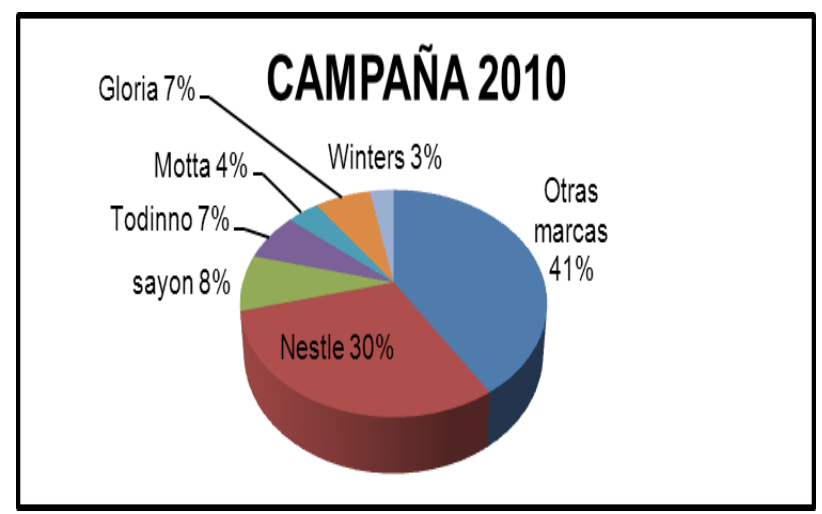

Figura 2. Mercado Nacional De Panetones Por Unidad Fuente: Latín panel 
Resultados del establecimiento de las ventas competitivas, productos y marcas como herramientas de gestión para el posicionamiento

\section{A. Establecimiento de las ventajas comparativas y competitivas}

Ventaja comparativa. Lima es un punto estratégico, por la cercanía de las empresas proveedoras de materia prima e insumos.

Ventaja competitiva. Habilidades para poder contrarrestar las limitaciones y producir el panetón a menor costo, minimizando el costo en todas las etapas del proceso de producción.

\section{B. Análisis del mercado de Estados Unidos}

La evolución de las importaciones de Estados Unidos, se muestra en Tabla 5.

Tabla 5. Comparativo Económico Perú - Estados Unidos

\begin{tabular}{ccc}
\hline Economía en comparación & $\begin{array}{c}\text { Estados } \\
\text { Unidos }\end{array}$ & Perú \\
\hline Población ( en millones) & 310 & 28.2 \\
PBI (en millones US\$) & 13,86 & $109,086.00$ \\
PBI per cápita (US\$) & $46,000.00$ & $3,865.00$ \\
Exportaciones de bienes y & 1.603 & 0.0297 \\
servicio (en Billones US\$) & & \\
$\begin{array}{c}\text { Importaciones de bienes y } \\
\text { servicios (en Billones US\$) }\end{array}$ & 1.05 & 0,026 \\
\hline
\end{tabular}

Fuente: http://www.indexmundi.com/es/estados unidos/

Resultados de la evaluación de las actividades productivas y comerciales de los productores

\section{A. Análisis de la cadena de valor}

\section{Logística de entrada}

Producción: Acondicionamiento de la planta industrial, condicionamiento y ubicación de las maquinarias.

Procesamiento: Recepción de materia prima (harina), compra de insumos.

\section{Operaciones}

Producción: Manejo agroindustrial del proceso, producto final.

Procesamiento: Embolsado, seleccionado, almacenado

\section{Logística de salida}

Producción: Venta en planta de Proceso, venta en mercados locales y nacionales, venta en ferias locales.

Procesamiento: Control y aseguramiento de la calidad, planificación de ventas, ventas en el mercado interno.

\section{Marketing y ventas}

Producción: Productor - consumidor, productor distribuidor, precios.

Procesamiento: Gestión de cliente, gestión de pedidos, canales de distribución, condiciones comerciales, Precio y publicidad.

\section{$\underline{\text { Servicios }}$}

Procesamiento: Asesoría en el consumo del producto.

\section{B. Análisis de la rentabilidad}

El costo de producción en planta se muestra en la Tabla 6.

Tabla 6. Costos de producción del Panetón.

\begin{tabular}{|c|c|c|c|}
\hline \multirow{2}{*}{ Insumos } & \multirow{2}{*}{$\begin{array}{c}\text { Precio } \\
\text { Unitario } \\
\text { S/ }\end{array}$} & \multicolumn{2}{|c|}{$\begin{array}{c}\text { Para } 1 \text { Saco de } 50 \\
\text { Kilogramos }\end{array}$} \\
\hline & & Materiales & Costo s/ \\
\hline \multicolumn{4}{|c|}{ Primer batido } \\
\hline Harina & 2 & $30 \mathrm{~kg}$ & 60 \\
\hline Azúcar & 2.5 & $7.5 \mathrm{~kg}$ & 18.75 \\
\hline Manteca & 4.4 & $5 \mathrm{~kg}$ & 15.75 \\
\hline Levadura & 9 & $1.5 \mathrm{~kg}$ & 13.5 \\
\hline Agua & 0.005 & $13 \mathrm{Lt}$ & 0.0065 \\
\hline Colorante & 0.05 & Al gusto & 0.05 \\
\hline Mejorador & 20 & $125 \mathrm{~g}$ & 2.5 \\
\hline Gluten & 9.5 & $500 \mathrm{~g}$ & 4.75 \\
\hline \multicolumn{4}{|c|}{ Segundo batido } \\
\hline Harina & 2 & $20 \mathrm{~kg}$ & 40 \\
\hline Azúcar & 2.5 & $8.5 \mathrm{~kg}$ & 21.25 \\
\hline Sal & 0.6 & $500 \mathrm{~g}$ & 0.3 \\
\hline Yemas de huevo & 9 & $8 \mathrm{~kg}$ & 72 \\
\hline Leche en polvo & 20 & $2 \mathrm{~kg}$ & 40 \\
\hline Antimoho & 11.5 & $150 \mathrm{~g}$ & 1.72 \\
\hline Mejorador & 20 & $125 \mathrm{~g}$ & 2.5 \\
\hline Agua & 0.005 & $7 \mathrm{Lt}$ & 0.0035 \\
\hline Lecitina & 8 & $750 \mathrm{~g}$ & 6 \\
\hline Pasas & 9 & $15 \mathrm{~kg}$ & 135 \\
\hline Fruta confitada & 6 & $15 \mathrm{~kg}$ & 90 \\
\hline Levadura & 9 & $500 \mathrm{~g}$ & 3.6 \\
\hline Emulsificante & 10 & $1 \mathrm{~kg}$ & 10 \\
\hline Primavera multiuso & 4.54 & $5 \mathrm{~kg}$ & 22.7 \\
\hline Esencia & 70 & $40 \mathrm{ml}$ & 28 \\
\hline Pirotines & 0.25 & 138 und & 34.5 \\
\hline Bolsas & 0.9 & 138 und & 124.2 \\
\hline Sellador & 0.01 & 138 und & 1.38 \\
\hline Caja de cartón & 2.7 & 25 unid & 67.5 \\
\hline Mano de obra & & destajo & 50 \\
\hline Energía & & & 20 \\
\hline Total & & & 885.96 \\
\hline
\end{tabular}

Fuente: Elaboración: Propia (2010)

Resultados de la evaluación de las encuestas y de la interrelación de variables propuestas

\section{Analisis de las encuestas}

De acuerdo a la operacionabilidad de variables, cada pegunta fue conducida con el fin de resolver las dudas planteadas que surgían en los diferentes grados de importancia, comose muestra en la Tabla 7 
Tabla 7. Encuesta Realizada

\section{Encuesta}

Pregunta 1: ¿De cuántos sacos de haría produce panetón al día?

$\begin{array}{cccccc}\text { Alternativas } & 1 \text { saco } & 5 \text { sacos } & 15 \text { sacos } & 20 \text { sacos } & \text { Más de } 20 \text { sacos } \\ \text { Resultados } \% & \mathbf{5} & \mathbf{1 5} & \mathbf{2 5} & \mathbf{2 8} & \mathbf{2 7}\end{array}$

Pregunta 2: ¿Qué nivel de tecnología utiliza para la producción de su panetón?
Alternativas
Bajo
Intermedio
Alto
Resultados \%
50
30
20

Pregunta 3: ¿Qué le falta para poder vender su panetón en el mercado internacional?
Alternativas
Fortalecer la empresa
Aumentar la
Nivel de
Capacidad de publicidad tecnología producción
Resultados \%

$13 \quad 15 \quad 30$
42

Pregunta 4: ¿se siente satisfecho con la cantidad de panetón que vende?
Alternativas
$\mathrm{Si}$
No
Resultados \%
38
62

Pregunta 5: ¿ha exportado alguna vez su panetón?
Alternativas
$\mathrm{Si}$
No
Resultados \%
30
70

Pregunta 6: ¿Le guitaría exportar el panetón que produce?
Alternativas
$\mathrm{Si}$
No
Resultados \%
98
2

Pregunta 7: ¿Ha participado de cursos de capacitación para la producción de panetón?
Alternativas
$\mathrm{Si}$
No
Resultados \%
33
67

Pregunta 8: ¿En qué presentación vende su panetón?
Alternativas
Bolsa
Cajas
Latas
Resultados \%
62
30
8

Pregunta 9: ¿Conoce las normas para comercializar panetón?
Alternativas
$\mathrm{Si}$
No
Resultados \%
26
64

Pregunta 10: ¿Dónde comercializa su panetón?

Alternativas

Resultados \%
Mercado internacional 14

Pregunta 11: ¿Qué característica le importa más de su panetón?

\begin{tabular}{cccc} 
Alternativas & sabor & Textura & tamaño \\
Resultados $\%$ & $\mathbf{4 5}$ & $\mathbf{4 0}$ & $\mathbf{1 5}$ \\
\hline
\end{tabular}

Fuente: Encuesta realizada (2011) 


\section{Analisis de la encuesta realizada}

1. Grado de importancia dada al análisis de la situación actual de los productores.

En la pregunta uno se obtiene los siguientes resultados, $5 \%$ producen un sacos, 15\% 5 sacos, 25\% 15 saco al día y $27 \%$ más de 20 sacos al día, demostrando que la mayoría de los empresarios producen de 10 a 20 sacos al día.

En la pregunta dos se obteniendo resultados que nos indican, que el $50 \%$ de productores utilizan tecnología baja, el 30 intermedia y el $20 \%$ alta, lo que demuestra que falta más tecnología en el Perú.

\section{Grado de importancia dada a la misión y visión.}

En la pregunta tres se analiza la misión y visión, obteniendo el siguiente resultado, el $13 \%$ responde fortalecer la empresa, $15 \%$ aumentar la publicidad, 30\% nivel tecnológico y el $42 \%$ capacidad de producción, demostrando que la mayor dificultad es la capacidad de producción.

\section{Grado de importancia dada al planteamiento de las estrategias}

En la pregunta cuatro se obtienen los siguientes resultados, el 30\% dice que sí y el 60 no, esto demuestra que falta un buen planeamiento para la exportación.

\section{Grado de importancia dada a los objetivos que se desea llegar.}

En la pregunta cinco se obtienen los siguientes resultados, $38 \%$ SI y $62 \%$ NO, demostrando que los productores tienen metas mayores.

\section{Grado de importancia dada al mercado del consumidor}

En la pregunta seis se obtienen los siguientes resultados, responde $98 \%$ SI y el 2\% NO, esto demuestra que a la mayoría le gusta exportar.

\section{Grado de importancia a la capacitación.}

En la pregunta siete se obtienen los siguientes resultados, el $33 \%$ dice SI y el $67 \%$ NO, esto indica que existe poca capacitación en pequeños empresarios.

\section{Grado de importancia dada al panetón.}

En la pregunta ocho se obtienen los siguientes resultados el $62 \%$ en bolsa polietileno, $30 \%$ en caja y el $8 \%$ en latas y otras presentaciones, esto nos muestra que el panetón de bolsa es más comercial.

\section{Grado de importancia dada al mercado meta.}

En la pregunta nueve se obtienen los siguientes resultados, el $26 \%$ dice que SI y el $74 \%$ NO, esto demuestra que hay poco conocimiento de las normas de comercialización

\section{Grado de importancia dada a la plaza.}

En la pregunta diez se obtienen los siguientes resultados, el $14 \%$ mercado nacional, $35 \%$ mercado de lima y el mercado nacional, esto indica que la exportación del panetón es mínima.

\section{Grado de importancia dada a las características del panetón.}

En la pregunta once se obtienen los siguientes resultados el $45 \%$ responden el sabor, $40 \%$ textura y el $15 \%$ tamaño, donde se observa que el sabor es la más importante.

\section{Plan de marketing}

El presente plan de marketing tiene como objetivo incentivar y diferenciar el panetón mediante el liderazgo de costos, minimizando los costos en todas las etapas del proceso productivo de las empresas panificadoras, en la exportación de panetón a Estados Unidos; este es un producto de panadería tradicional en la cena navideña peruana y perteneciente a la industria manufacturera alimentaria peruana.

Su origen se remonta a Milán (Italia), pero su elaboración y consumo se ha expandido a lo largo del tiempo adoptando diferentes nombres y formas de elaboración en las distintas partes del globo.

El consumo nacional se realiza principalmente en navidad y fiestas patrias; lo mismo ocurre en el mercado internacional, debido a la presencia de gran cantidad de peruanos en los principales mercados de destino como Venezuela, donde son más de 100 mil los peruanos que allí residen, constituyéndose en el primer destino de nuestra exportaciones hasta el año 2009, ahora EE.UU ocupa el primer lugar esto gracias por el Tratado de Libre Comercio.

El plan de marketing para el panetón tiene como propósito diferenciar sus productos e ingresar a un nuevo mercado, por lo tanto su estrategia es la de penetración, respaldada con la diferenciación del producto, y como estrategia genérica se empleará la de liderazgo en costos, puesto que en el mercado de Estados Unidos la variación de precios es inestable y se tiene la posibilidad de ingresar a costos menores. Los objetivos estratégicos están orientados hacia la comercialización mediante las ventas, posicionamiento y rentabilidad, asimismo, se establecen objetivos de distribución enfocados a la publicidad, comercialización y mercado meta objetivo.

Finalmente, los criterios de inversión afirma una tasa interna de retorno del $21 \%$ calculando para una producción de un saco de harina, el valor actual neto de S/ 518.00, los indicadores financieros son positivos y estos aseveran la viabilidad del proyecto. 


\section{A. Análisis del entorno}

\section{a. Análisis del entorno general}

La evolución de las exportaciones del panetón se ha incrementado a partir del año 2006 y es en el año (2010), donde la variación porcentual se ha incrementado hasta en $38 \%$ en cuanto al valor FOB y en cuanto al volumen hasta en $(24 \%)$.

De los mercados a donde se ha dirigido el panetón durante los últimos años, son Estados Unidos, Venezuela, Bolivia, Ecuador y Chile, los principales compradores, para la exportación de los panetones en las diferentes presentaciones; son productos que tienen mayor valor agregado, donde la combinación de trabajo y tecnología generan una categoría nueva para el mismo producto.

\section{b. Análisis del entorno específico}

De acuerdo al análisis de las cinco fuerzas competitivas de Michael Porter anteriormente realizado se considera el siguiente entorno específico:

Considerando que la economía del mundo está globalizada e impera el libre mercado, donde no existen barreras de entrada y salida para el sector de panificación de Lima metropolitana por eso el incremento de empresas panificadoras, en su mayoría informales.

\section{B. Análisis interno}

a. La empresa. Las panificadoras están constituidos legalmente, pero existe el $40 \%$ de panaderías informales, dentro de todos que producen panetón, son empresas que tienen muchos años en el mercado nacional y producen muchas clases de productos dentro de la panadería y son quienes se encargan de gestionar la comercialización a través de todo los canales de distribución.

b. Análisis FODA. El análisis FODA determina la situación de la empresa en el entorno las oportunidades y amenazas y en interno las fortalezas y debilidades.

\section{Oportunidades}

Apertura económica con el Tratado de Libre Comercio.

Reducido número de países productores.

Posibilidad de expansión del mercado actual.

Tamaño potencial de mercado nacional e internacional.

Existencia de alta demanda mundial.

Políticas gubernamentales favorables para la exportación.

Presión atmosférica y clima favorable para su producción.

\section{Amenazas}

Bajo nivel de tecnología a comparación de otros países.

Requerimiento de alta inversión para competir.

Inestabilidad en precios internacionales de materia prima.

Poco apoyo para las investigaciones del panetón.

Alta inestabilidad en el comportamiento del mercado exterior.

\section{Fortalezas}

Bajo costo de mano de obra en del proceso de producción Alta rentabilidad obtenida.

Calidad del producto con buen tiempo de vida útil.

Características nutricionales importantes.

Producto con alto porcentaje de ingresos.

\section{Debilidades}

Falta mayor organización de los empresarios productoras.

Plantas procesadoras en su mayoría pequeñas.

Utilización de una tecnología intermedia.

Parcial identificación de los productores con el mercado externo.

Pocas empresas tienen certificaciones de calidad.

Competencia desleal de los empresarios productores.

\section{c. La misión y Visión}

Misión.- Producir y comercializar para satisfacer las necesidades del consumidor y mejorar las condiciones de vida de las pequeñas o medianas empresas.

Visión.-Ser empresa líder y competitiva en base o costos, sabor y duración, para el mercado internacional.

\section{d. Los objetivos}

\section{Objetivo general}

Introducir el producto en el mercado de California, para captar el $10 \%$ de la cuota de mercado.

\section{Objetivos específicos}

Incrementar el volumen de ventas y obtener una rentabilidad de $30 \%$ al término del segundo periodo de exportación.

Posicionar el producto de panetón bajo la marca RICOSON.

e. La estrategia genérica. Fortalecimiento de la fuerza de ventas mediante el incremento en el número empresas productoras.

f. La estrategia de entrada. Por medio de una venta estratégica por costos bajos y calidad.

\section{La investigación de mercado}

\section{- Objetivos de la investigación}

Determinar el mercado al cual se ingresará el panetón como un producto industrial.

\section{- Fuentes de información secundaria}

PROMPERU,Asociación de Exportadores, Universidades, Instituto Nacional de Estadística e Informática.

\section{- Pronóstico de la Oferta y Demanda}

Según los datos obtenidos por la SUNAT; en el año 2010 el Perú exportó un valor FOB de USS 4,836 millones, correspondiente a un volumen de 1,518.74 TM.

\section{- Medida del Tamaño de Mercado}

Se refiere al número de compradores que pueden existir 
para la oferta determinada, es posible identificar las cuatro categorías siguientes, como se observa en la fig. 4 .

Figura 4. Tamaño del mercado.

Población total de California 38 millones de habitantesLos Ángeles 3,792.621 de habitantes

\begin{tabular}{|c|}
\hline \multicolumn{1}{|c|}{$\begin{array}{c}\text { Mercado potencial } 46 \% \text { de los habitantes } \\
\text { hispanos } 1,764.706 \text { habitantes }\end{array}$} \\
\hline $\begin{array}{c}\text { Mercado objetivo } 20 \% \text { del mercado potencial } \\
352.941 \text { consumidores }\end{array}$ \\
\hline $\begin{array}{c}\text { Mercado actual } 5 \% \text { del mercado objetivo } \\
17.647 \text { consumidores }\end{array}$ \\
\hline
\end{tabular}

Fuente: Modelo de Plan de Marketing Mayorga y Araujo 2011, Elaboración propia

\section{La formulación estratégica de marketing}

a. El análisis del mercado. El análisis del mercado, cultural, externo e interno se muestra en los Tablas 8,9, 10, 11 y 12 .

Tabla 8. Factores Externos. La cultura

\begin{tabular}{cc}
\hline Nacionalidad & Americanos, latinos \\
Religión & Cristiana protestante \\
Región & California - Los Ángeles \\
Raza & Blancos no hispanos, hispanos, \\
latinos \\
Rango de edad & De cinco años a más \\
Sexo & Hombres y mujeres \\
Ocupación & Estudiantes, profesionales y amas \\
de casa
\end{tabular}

Fuente: Elaboración: Propia (2011)

Tabla 9. Factor Externo, Clase Social.

\begin{tabular}{ll}
\hline & Alto \\
Clase social California - & Medio \\
Los Ángeles & Bajo \\
\hline
\end{tabular}

Fuente: Elaboración Propia (2011)

Tabla10. Factor Interno, La Motivación.

\begin{tabular}{ll}
\hline $\begin{array}{l}\text { Motivos } \\
\text { compra }\end{array}$ de $\begin{array}{l}\text { Significado de amor y amistad del } \\
\text { producto, sabor agradable, tamaño, } \\
\text { colory textura, que resulta atractivo }\end{array}$ \\
\hline
\end{tabular}

Fuente: Elaboración Propia (2011)
Tabla 11. Factor Interno, la Percepción.

\begin{tabular}{cl}
\hline Amas de & Producto especial con mucho significado \\
casa & de amor y amistad. \\
Niños & Agradable \\
Jóvenes & Sabor para compartir en amor y amistad \\
\hline
\end{tabular}

Fuente: Elaboración: Propia (2011)

Tabla 12. Factor Interno, la Personalidad.

\begin{tabular}{c} 
Niños de kínder, jóvenes, personas \\
Personalidad $\begin{array}{c}\text { maduras, satisfechas y reflexivas que } \\
\text { son motivados por productos que } \\
\text { significan amistad y amor. }\end{array}$ \\
\hline Fuente: Elaboración: Propia (2011)
\end{tabular}

b. Las estrategias de marketing: Segmentación: conductual y psicográficas.

\section{E. La mezcla de marketing}

Producto: Variedad, calidad, características, marca, envase.

Precio: Lista de precios, descuentos.

Plaza: Cobertura, surtido, inventario.

Promoción: Todas las publicidades necesarias.

\section{F. El análisis económico}

\section{Los supuestos}

Periodo de evaluación: El proyecto de desarrollo para un periodo de exportación y un análisis de diez años.

Moneda: se establece en dólares americanos al tipo de cambio actual S/ 2.80 .

\section{Calculo de precio de exportación}

En la Tabla 13, se tiene el cálculo del precio de exportación, considerando todo los gastos necesarios y teniendo un resumen de los diferentes valores obtenidos para cada INCOTERM.

Tabla 13. Cálculo del precio de exportación/TM

\begin{tabular}{lcc}
\hline \multicolumn{1}{c}{ Descripción del elemento } & Importe & \% \\
\hline Costo variable Bruto del Producto (CVB) & \\
Costo de materiales & 2111.7 & $34.40 \%$ \\
Costo de mano de Obra Directa & 129.4 & $2.10 \%$ \\
Costo Directo de Producción & 569.4 & $9.30 \%$ \\
Total Costos Variables Bruto & 2810.4 & $45.80 \%$ \\
Costo Variable Neto del Producto (CVN) & \\
Devolución de Impuestos y Tasas & 0 & $0.00 \%$ \\
(Drawbacks) & 2810.4 & $45.80 \%$ \\
Total de costos Variable Neto & & \\
Margen de Contribución (MC) & 831 & $13.50 \%$ \\
\hline
\end{tabular}


Continuación de tabla 13

\begin{tabular}{lcc}
\hline Total de Precio (CVN + MC) & 3641.4 & $59.30 \%$ \\
Valor EX Works (VEW) & & \\
Costos Seguro de Cambio & 0 & $0.00 \%$ \\
Costos Seguro de Crédito & 0 & $0.00 \%$ \\
$\begin{array}{l}\text { Gastos Financieros Aplazamiento } \\
\text { de Pago }\end{array}$ & 0 & $0.00 \%$ \\
Comisión de Agente & 150 & $2.40 \%$ \\
Costos de embalajes & 110 & $1.80 \%$ \\
$\begin{array}{l}\text { Costos Documentación de } \\
\text { exportación }\end{array}$ & 35.7 & $0.60 \%$ \\
$\begin{array}{l}\text { Costos Adecuación Producto al } \\
\text { Mercado Destino } \\
\text { Total Costos Específicos Ex }\end{array}$ & 0 & $0.00 \%$ \\
Works & 295.7 & $4.80 \%$ \\
$\begin{array}{l}\text { Motal Valor ExWorks (CVN + VEW) } \\
\text { Valor FAS (Franco al Costado) }\end{array}$ & 3937.1 & $64.10 \%$ \\
Costos de Manipulación & & \\
Costos Transporte Interno & 32.1 & $0.50 \%$ \\
$\begin{array}{l}\text { Gastos/Trámites aduaneros } \\
\text { Exportación } \\
\text { Total Valor FAS }\end{array}$ & 107.1 & $1.70 \%$ \\
Valor FOB/FOT/FOR (Fran & 166.1 & $2.70 \%$ \\
& 4242.5 & $69.10 \%$
\end{tabular}

Valor FOB/FOT/FOR (Franco a bordo Camión/tren)

$\begin{array}{lcc}\text { Costos carga mercancía } & 28.6 & 0.50 \% \\ \text { Costos Tasa Puerto de Salida } & 14 & 0.20 \% \\ \text { Total Valor FOB } & 4285.1 & 69.80 \%\end{array}$

Puerto de Origen:

Valor CIF (Costos, Seguro y Flete)

Costos Transporte Internacional

Costos Seguro

Total Precio CIF

Puerto/Lugar de Desembarque:

Precio Venta al Importador (DDP)

Aranceles y tasas

Gastos Portuarios

Gastos Transporte en Destino

Total Precio Venta Importador (DDP)

Precio Venta al Minorista

Recargo

Margen Mayorista

Total Precio Venta al Minorista

PVP Previo

Recargo

Margen Detallista

$8 \%$

\section{Total PVP Previo}

Precio de venta final público

\begin{tabular}{lcc} 
Ajustes de Competencia & 200 & $3.30 \%$ \\
Margen de Maniobra & 200 & $3.30 \%$ \\
$\begin{array}{lcc}\text { Total Precio de Venta Final } \\
\text { Público }\end{array}$ & $\mathbf{6 1 3 7 . 9}$ & $\mathbf{1 0 0 \%}$ \\
\hline
\end{tabular}

Elaboración: Propia (2011)

$180 \quad 2.90 \%$

$64.7 \quad 1.10 \%$

$\mathbf{4 5 2 9 . 7} \quad 73.80 \%$

\section{Conclusiones}

El plan de marketing elaborado para el panetón, con el objetivo de exportar a Estados Unido, influye el incremento de las ventas.

El panetón producido en Lima, si puede satisfacer la demanda del mercado Norteamérica, debido a la cantidad de producción del panetón.

Las ventajas competitivas en base a calidad del panetón y las ventajas competitivas en base a costos, influyen en la intención de posicionar en el mercado internacional.

La realidad de la actividad industrial y comercial, permite lograr una rentabilidad aproximado de $21 \%$, considerando que sus egresos son bajos y los ingresos son altos.

\section{Recomendaciones}

Ejecutar programas de asistencia técnica a los pequeños empresarios, para optimizar el desarrollo de la producción de acuerdo a sus requerimientos.

Establecer el panetón como un producto natural, nutricional con certificaciones.

Evaluar los costos de producción del panetón de los países competidores.

Estudiar todas las presentaciones del panetón para nuevos mercados.

\section{Literatura citada}

Ampex. 2010. Asociación Macroregional de Productos Para la Explotación. Edición 2010. 4 P.

Howyhon, J. 1983. Fundamento de las ciencias, los alimentos. Zaragoza, España. 18 P.

Kotler, P. 1993. Dirección de mercadotecnia. Prentice. Hall. México. 695 P.

Mayorga, D y Araujo P. 2010. El plan de Marketing. Centro de investigación. Universidad del pacifico Lima. Lima, Perú. 134 P.

Meyer HG. y Tobin G. 1991. Nutrición y ciencia se los alimentos Editorial Acriba. Zaragoza, España. 28 P.

$393.5 \quad 6.40 \% \quad$ Masson JE. y Wellhoff A. 1990 "El Merchandising. 5312.9 $86.60 \%$ Rentabilidad y gestión del punto de venta. Bilbao. Editorial Deusto.254 P.

Muñiz G, R. 2003."Marketing del siglo XXI". Políticas de precios. Consultado el 27 de agosto del 2010.

Poter, M. 2006, Estrategia y ventaja comparativa, Colombia $57 \mathrm{p}$. 